\title{
Extent of Late Gadolinium Enhancement Predicts Thromboembolic Events in Patients With Hypertrophic Cardiomyopathy
}

\author{
Anna Hohneck, MD; Daniel Overhoff, MD; Christina Doesch, MD; \\ Raphael Sandberg, MD; Boris Rudic, MD; Erol Tueluemen, MD; Johannes Budjan, MD; \\ Kristina Szabo, MD; Martin Borggrefe, MD; Theano Papavassiliu, MD
}

\begin{abstract}
Background: Thromboembolic complications such as ischemic stroke or peripheral arterial thromboembolism are known complications in hypertrophic cardiomyopathy ( $\mathrm{HCM})$. We sought to assess the clinical and cardiovascular magnetic resonance $(\mathrm{CMR})$ characteristics of patients with HCM suffering from thromboembolic events and analyzed the predictors of these unfavorable outcomes.

Methods and Results: The $115 \mathrm{HCM}$ patients underwent late gadolinium enhanced (LGE) CMR and were included in the study. Follow-up was $5.6 \pm 3.6$ years. The primary endpoint was the occurrence of thromboembolic events (ischemic stroke or peripheral arterial thromboembolism). It occurred in 17 (14.8\%) patients (event group, EG), of whom 64.7\% (11) were men. During follow-up, $10(8.7 \%)$ patients died. Patients in the EG showed more comorbidities, such as heart failure (EG $41.2 \%$ vs. NEG (non-event group) $14.3 \%, P<0.01$ ) and atrial fibrillation (AF: $E G 70.6 \%$ vs. NEG 36.7\%, $P<0.01$ ). Left atrial end-diastolic volume was significantly higher in the $E G$ ( $E G$ 73 \pm 24 vs. NEG $50 \pm 33 \mathrm{~mL} / \mathrm{m}^{2}, P<0.01$ ). Both the presence and extent of $L G E$ were enhanced in the $E G$ (extent\% EG $23 \pm 15 \%$ vs. NEG $8 \pm 9 \%, P<0.0001$ ). No patient without $L G E$ experienced a thromboembolic event. Multivariate analysis revealed $\mathrm{AF}$ and LGE extent as independent predictors.
\end{abstract}

Conclusions: LGE extent (>14.4\%) is an independent predictor for thromboembolic complications in patients with HCM and might therefore be considered as an important risk marker. The risk for thromboembolic events is significantly elevated if accompanied by AF.

Key Words: Atrial fibrillation; Hypertrophic cardiomyopathy; Late gadolinium enhancement; Thromboembolic event

H ypertrophic cardiomyopathy (HCM) is a complex genetic heart disease ${ }^{1-4}$ that is defined by the presence of increased myocardial wall thickness not resulting from abnormal loading conditions. ${ }^{5}$ Thromboembolic events such as ischemic stroke and peripheral arterial thromboembolism are known to occur as complications of HCM. ${ }^{68}$

Atrial fibrillation (AF) is the most common arrhythmia in patients with $\mathrm{HCM} .{ }^{9}$ As patients with $\mathrm{HCM}$ tend to be younger at the onset of $\mathrm{AF},{ }^{10}$ and in view of the high incidence of thromboembolic complications in this cohort, ${ }^{11}$ estimating the risk by calculating the $\mathrm{CHA}_{2} \mathrm{DS}_{2}$-VAScScore does not seem to be appropriate. ${ }^{12}$ Therefore, all patients with HCM and AF (paroxysmal, persistent or permanent) should receive treatment with vitamin $\mathrm{K}$ antagonist (VKA), which was adopted in 2014 as a Class I

\begin{abstract}
Editorial p 700
recommendation, level $\mathrm{B}$ of evidence into the European Society of Cardiology (ESC) Guidelines on diagnosis and management of HCM. ${ }^{13}$

The association between late gadolinium enhanced (LGE) and long-term outcomes has been examined in several studies, revealing a relationship between LGE and cardiovascular death or heart failure-related death. ${ }^{14-18}$ Furthermore, patients with HCM and a high ESC risk score reveal a higher extent of LGE, which supports the role of LGE as an additional risk marker. ${ }^{19}$

The incidence of ischemic stroke and peripheral arterial thromboembolism is markedly increased if complicated by AF in patients with HCM. ${ }^{7}$ Therefore this study analyzing
\end{abstract}

Received October 25, 2019; revised manuscript received February 12, 2020; accepted February 18, 2020; J-STAGE Advance Publication released online April 1,2020 Time for primary review: 26 days

1st Department of Medicine Cardiology (A.H., C.D., R.S., B.R., E.T., M.B., T.P.), DZHK (German Center for Cardiovascular Research) partner site (A.H., B.R., E.T., M.B., T.P.), Institute of Clinical Radiology and Nuclear Medicine (D.O., J.B.), and Department of Neurology (K.S.), University Medical Center Mannheim, Medical Faculty Mannheim, University of Heidelberg, Mannheim, Germany

The first two authors contributed equally to this work (A.H., D.O.).

Mailing address: Anna Hohneck, MD, University Medical Center Mannheim, First Department of Medicine - Cardiology, Theodor-Kutzer-Ufer 1-3, DE-68167 Mannheim, Germany. E-mail: annalena.hohneck@umm.de

ISSN-1346-9843 All rights are reserved to the Japanese Circulation Society. For permissions, please e-mail: cj@j-circ.or.jp 
clinical and cardiovascular magnetic resonance (CMR) characteristics was performed to ascertain the predictors for these unfavorable events in a HCM cohort.

\section{Methods}

\section{Study Population}

A total of 115 patients from our HCM clinic who underwent physical examination, transthoracic echocardiography (TTE), CMR examination and serial 48-h Holter monitoring between January 2004 until December 2012 were included in the study and followed prospectively. Mean follow-up time was $5.6 \pm 3.6$ years. A part of this study was reported earlier in another context. ${ }^{19}$

Inclusion criteria were patients with $\mathrm{HCM}$, with the diagnosis based on conventional criteria; left ventricular (LV) hypertrophy, $>15 \mathrm{~mm}$ on 2D echocardiography in the absence of another disease that could account for the hypertrophy. Nonobstructive HCM was defined as a pressure gradient $\leq 30 \mathrm{mmHg}$ at rest and after provocation. Patients with a pressure gradient $>30 \mathrm{mmHg}$ at rest or after provocation were classified as obstructive $\mathrm{HCM}^{20}$

Exclusion criteria were contraindications for CMR (metallic implant or device, such as pacemaker or implanted cardioverter-defibrillator), claustrophobia and renal dysfunction (estimated glomerular filtration rate $\leq 30 \mathrm{~mL} /$ $\min / 1.73 \mathrm{~m}^{2}$ ), acute myocardial infarction and previous history of thromboembolic events.

CMR and TTE were performed at the time of study enrolment.

The study design complied with the Declaration of Helsinki and was approved by the Ethical Committee, Medical Ethic Commission II, Faculty of Medicine Mannheim, University of Heidelberg, Germany. Written informed consent was obtained from all patients at the time of CMR examination and data were analyzed anonymously.

\section{CMR Image Acquisition}

All studies were performed using a 1.5-Tesla whole body imaging system (Magnetom Avanto or Sonata, Siemens Medical Systems, Healthcare Sector, Erlangen, Germany) using a 4-element (Sonata) or a 6-element (Avanto), phased-array body coil.

Cine images were acquired using a retrospective ECGgated, balanced segmented steady state free precession (trueFISP) sequence in 3 long-axis views (2-, 3-, and 4-chamber view) and in multiple short-axis views, covering the entire LV from base to apex.

LGE images were obtained $10-15 \mathrm{~min}$ after intravenous administration of $0.2 \mathrm{mmol} / \mathrm{kg}$ gadoteric acid (Dotarem, Guerbet, Germany), using an inversion recovery turbo fast low angle shot sequence at the same position as the long- and short-axis cine acquisitions in end-diastole. ${ }^{21}$ The inversion time was adjusted per patient to optimally null the signal from normal myocardium, which is typically between 250 and $300 \mathrm{~ms}$.

\section{CMR Image Analysis}

$\mathrm{LV}$ mass and volumes, ${ }^{22}$ as well as right ventricular $(\mathrm{RV})^{23}$ and left atrial (LA) volumes, ${ }^{24}$ were determined using CMR as previously described. The LV remodeling index (LVRI) was determined as the ratio of LV mass to LV end-diastolic volume. ${ }^{25}$ LA volumes were assessed using the biplane area-length method, which can be also performed in patients with varying cardiac cycle lengths, as in AF. ${ }^{24}$
MAPSE measurements were assessed on 4-chamber view cine images. The distance between the basal septal mitral annulus (septal MAPSE), the basal lateral mitral annulus (lateral MAPSE) and a reference point outside the LV apex was measured in end-diastole (EDL) and end-systole (ESL). Average MAPSE was calculated as the average of septal and lateral MAPSE. ${ }^{\mathbf{2 6}}$

To determine TAPSE, the distance between the cutting edge of the tricuspid annulus with the RV free wall and a reference point outside the RV apex was measured in EDL and ESL. The point outside the RV apex was chosen as extension to the RV apex and had to stay unchanged at EDL and ESL. TAPSE was defined as the difference between EDL and ESL. ${ }^{26,27}$

\section{LGE Quantification}

The extent of LGE was assessed by a semiquantitative score based on the standard LV 17-segment model, as described previously. ${ }^{28,29}$ Each segment was visually assessed and scored by 2 experienced observers (C.D. and D.O.) for the distribution and the total extent of LGE was expressed as a percentage of the LV myocardial area, blinded to clinical data.

\section{Reproducibility}

For intraobserver variability, observer 1 (C.D.) performed LGE analyses for all subjects $(n=115)$, with a second analysis after 30 days. For interobserver variability, observer 2 (D.O.) independently also assessed LGE for all subjects. Intra- and interobserver agreements for LGE analyses were determined using intraclass correlation coefficient (ICC).

\section{TTE}

TTE was performed in the supine/left lateral decubitus position with a phased-array ultrasound sector scanner (Vivid E9, GE Healthcare, UK) and a 3.5-MHz transducer. The 2D images were obtained in the standard parasternal and apical views. All Doppler recordings were obtained with a sweep speed of $100 \mathrm{~mm} / \mathrm{s}$.

\section{Follow-up Data and Definition of Study Endpoint}

Long-term follow-up (5.6 \pm 3.6 years) was performed within the routine visits to the HCM clinic or by telephone contact for patients being treated by another physician. Reported clinical events were confirmed by review of the corresponding medical records in the electronic hospital information system, or contact with the general practitioner, referring cardiologist, or treating hospital. The observer was unaware of the CMR results and collected data with a standardized questionnaire. The occurrence of thromboembolic events was defined as the primary endpoint. Ischemic stroke was defined as neurological deficit of vascular cause lasting $>24 \mathrm{~h}$ or ischemic event lasting $<24 \mathrm{~h}$ with imaging evidence of stroke. ${ }^{30}$ Cerebral perfusion imaging was performed in every patient (computed tomography or magnetic resonance) to confirm the diagnosis and to exclude hemorrhage. The diagnosis of peripheral arterial thromboembolism (lower limb arteries, mesenteric or renal artery) was based on Doppler sonography or arteriography.

\section{AF}

The presence of AF was based on ECG evidence, including Holter monitoring, which was performed at least twice per year. Classification of AF was performed in accordance 
with current ESC guidelines for the management of AF, ${ }^{12}$ in which 2 patterns, paroxysmal and permanent, are differentiated.

\section{Statistical Analysis}

Data are presented as mean \pm standard deviation, or frequency (percentage). Continuous variables were compared using a 2-tailed Student's t-test for parametric and MannWhitney $\mathrm{U}$ test for non-parametric variables. Categorical variables were compared with the $\chi^{2}$ test.

Univariate analysis was performed by linear regression to identify predictors associated with the occurrence of thromboembolic events. A Chi-square test was used to evaluate if the presence of LGE was different between groups. The ICC were calculated for the assessment of both intra- and interobserver agreement for LGE assessment, providing a coefficient ranging from 0 to 1 and its 95 confidence interval $(\mathrm{CI})$, with a high ICC close to 1 indicating high similarity.

Multivariate analysis was performed with logistic regression analysis using block entry of the following variables: LGE extent, LA end-diastolic volume (LAEDV) and presence of $\mathrm{AF}$, proved to have $\mathrm{P}<0.01$ in the univariate analysis.

Receiver-operating characteristic (ROC) curves were used in the whole group of patients to find the optimal cutoff value for LGE extent (maximizing the sum of sensitivity and specificity).

Based on the results of the multivariate analysis and the ROC curve, a risk model was established, estimated with the Kaplan-Meier method and compared with the log-rank test.

All results were considered statistically significant when $\mathrm{P}<0.05$. Analyses were performed with Statistical 1 Package for Social Sciences (SPSS for Windows 14.0, Chicago, IL, USA) and GraphPad Prism 7.0 (Graphpad Software, Inc., CA, USA).

\section{Results}

The mean age of the study population was $55.3 \pm 15.2$ years. There was a trend to older patients in the event group (EG)

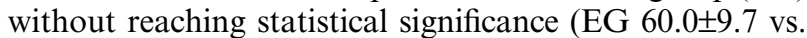
non-event group (NEG) 54.5 $\pm 15.9, \mathrm{P}=0.17$ ). Two-thirds of the study population were male (EG $11(64.7 \%)$ vs. NEG $62(62.3 \%), \mathrm{P}=0.91)$. The nonobstructive phenotype of HCM was more common (EG $12(70.6 \%)$ vs. NEG 62 $(63.3 \%), \mathrm{P}=0.56)$. Severe concomitant diseases such as heart failure (NYHA III-IV) were found more often in the EG group (EG 7 (41.2\%) vs. NEG 14 (14.3\%), P<0.01). A total of $10(8.7 \%)$ patients died during the follow-up period of 5.6 \pm 3.6 years (Supplementary Table).

With regard to medications, 15 patients (EG $5(29.4 \%)$ vs. NEG $10(10.2 \%), \mathrm{P}=0.03)$ received amiodarone, and 3 patients in the NEG received VKA for other indications (ventricular thrombus, patent foramen ovale, mechanical valve replacement).

A total of $28(24.4 \%)$ of 118 patients suffered from AF before CMR examination. At the time of CMR examination, $9(7.8 \%)$ patients presented with permanent AF (EG 4 (23.5) vs. NEG 5 (5.1), $\mathrm{P}<0.01$ ); the remaining study population showed sinus rhythm. Of the 28 patients with AF, $21(75.0 \%)$ were receiving anticoagulant agents such as VKA (EG 5 (29.4) vs. NEG 12 (12.2)) or direct oral anticoagulants (DOAC) (EG 1 (5.9) vs. NEG 3 (3.1)).
Serial Holter monitoring was performed during follow-up (at least twice per year) in order to screen for AF. During follow-up, 22 (19.1\%) additional patients were diagnosed with AF (EG $4(23.5 \%)$ vs. NEG 18 (18.4\%), P=0.62). Of them, $18(81.8 \%)$ were taking anticoagulant agents such as VKA (EG $2(11.8 \%)$ vs. NEG $9(9.2 \%)$ ) or DOAC (EG 2 $(11.8 \%)$ vs. NEG $5(5.1 \%))$. The overall presence of AF (AF before CMR and development during follow-up) was significantly higher in the EG group (EG $13(76.5 \%)$ vs. NEG $37(32.2 \%), \mathrm{P}<0.01)$.

The primary endpoint occurred in $17(14.8 \%)$ patients: 11 male and 6 female; 16 patients suffered an ischemic stroke and 1 had peripheral arterial embolism. Mean age at the time of the event was $62.6 \pm 10.6(64.1 \pm 11.3)$ years. $\mathrm{AF}$ was present in 9 patients before $\mathrm{CMR}$, and was the permanent type in 4 cases. Of these 9 patients, 7 (77.8\%) received anticoagulant therapy (VKA or DOAC). The remaining 2 patients were treated with acetylsalicylic acid (ASA) alone or dual antiplatelet therapy (DAPT). During follow-up 4 additional patients were diagnosed with AF: anticoagulant therapy was initiated in 3 patients $(75.0 \%)$, but was declined by 1 patient.

In 3 cases thromboembolic events were most probably due to insufficient doses of VKA or DOAC, 3 other patients were treated ineffectively with platelet inhibition (ASA, clopidogrel) and 1 patient developed stroke during bridging with low-molecular-weight heparin.

In our cohort, $\mathrm{AF}$ could be documented in 13 of the 17 patients in the EG and identified as causative for the thromboembolic event. In $4(23.5 \%)$ patients there was no detectable AF or known history, which is why the event was diagnosed as "cryptogenic". However, paroxysmal AF could not be ruled out with absolute certainty. Additionally, LV aneurysms were excluded carefully in these 4 patients, as this phenotype is also associated with a higher risk for thromboembolic events.

There was a trend to a decreased LV ejection fraction (EF; within the normal range) in the EG group (EG 55 \pm 10 vs. NEG $61 \pm 10, P=0.02$ ). Average MAPSE was also reduced (EG $0.76 \pm 0.38 \mathrm{~cm}$ vs. NEG $1.07 \pm 0.58 \mathrm{~cm}, \mathrm{P}=0.04$ ), but $\mathrm{LV}$ volumes or septal and posterior wall dimensions were comparable in both groups.

Furthermore, patients who suffered a thromboembolic event revealed a significantly reduced RVEF, also within the normal range (EG $56 \pm 11 \%$ vs. NEG $62 \pm 10 \%, \mathrm{P}=0.03$ ) and TAPSE (EG $1.45 \pm 0.57 \mathrm{~cm}$ vs. NEG $1.79 \pm 0.51 \mathrm{~cm}$, $\mathrm{P}=0.02$ ), with enlargement of the RA ( $\mathrm{EG} 48 \pm 3 \mathrm{~mm}$ vs. NEG $43 \pm 7 \mathrm{~mm}, \mathrm{P}=0.03$ ).

Both the presence (EG $17(100.0 \%)$ vs. NEG $62(63.3 \%)$, $\mathrm{P}<0.01$ ) and extent (EG $23 \pm 15 \%$ vs. NEG $8 \pm 9 \%, \mathrm{P}<0.0001$ ) of LGE were significantly higher in the EG group. Importantly, in our population, no patient without detectable fibrosis experienced a thromboembolic event.

LAEDV was significantly increased (EG $73 \pm 24$ vs. NEG $\left.50 \pm 33 \mathrm{~mL} / \mathrm{m}^{2}, \mathrm{P}<0.01\right)$ compared with the patients with HCM without thromboembolic events.

ICC were calculated for the assessment of both intra- and interobserver agreement for LGE assessment with excellent to nearly perfect agreement for interobserver (ICC 0.88 [0.70-0.95]) and intraobserver agreement (ICC 0.92 [0.81$0.96])$.

Patient demographics, echocardiographic parameters, AF characteristics and CMR parameters are outlined in Table 1. Multivariate analysis was performed with logistic regression analysis using block entry of the following 
Table 1. Patients' Demographics, Echocardiographic Parameters, AF Characteristics and CMR Parameters

\section{Age (years) at time of CMR \\ Male, $\mathbf{n}(\%)$ \\ Death during follow-up, $\mathrm{n}(\%)$ \\ Hypertrophic cardiomyopathy, $\mathrm{n}(\%)$ \\ HOCM \\ HNCM \\ Apical aneurysm \\ Palpitations \\ Syncope \\ Positive family history for SCD \\ Comorbidities, $\mathbf{n}(\%)$ \\ Hypertension \\ History of smoking \\ Hyperlipidemia \\ Diabetes mellitus \\ $\mathrm{CHA}_{2} \mathrm{DS}_{2}$-Vasc-Score \\ NYHA III+IV \\ GFR $<60 \mathrm{~mL} / \mathrm{min} / 1.73 \mathrm{~m}^{2}$}

$\geq$ Moderate $\mathrm{Ml}$

Concomitant medications at time of CMR, $\mathbf{n}(\%)$

$\beta$-blocker

Calcium-channel blocker

Amiodarone

ASA

Clopidogrel

VKA (other indication than AF)

Echocardiographic parameters

LVEF (\%)

LVH distribution, $n(\%)$

Anterior septum

Anterior and posterior septa

Both septa and free wall of LV

LVEDD (mm)

LVOT obstruction $(\mathrm{mmHg})$

Diastolic dysfunction, ${ }^{\mathrm{A}} \mathrm{n}$ (\%)

E-wave (ms)

E/E'

$\operatorname{TR} \operatorname{Vmax}(\mathrm{m} / \mathrm{s})$

LA M-Mode $(\mathrm{mm})$

LAVI $\left(\mathrm{mL} / \mathrm{m}^{2}\right)$

AF characteristics

AF before CMR, $n(\%)$

Proportion of permanent AF

Anticoagulant therapy before CMR, $\mathrm{n}(\%)$

VKA

Dabigatran

Rivaroxaban

Apixaban

AF during follow-up, $n$ (\%)

Anticoagulant therapy during follow-up, $n(\%)$

VKA

Dabigatran

Rivaroxaban

Apixaban
All patients with $\mathrm{HCM}$ $(n=115)$

$55.3 \pm 15.2$

$73(63.5)$

$10(8.7)$

41 (35.7)

$74(64.4)$

5 (4.3)

$46(40.0)$

$31(27.0)$

$18(15.7)$

15 (13.0)

$25(21.7)$

$33(28.7)$

$9(7.8)$

$1.2 \pm 1.3$

21 (18.3)

14 (12.2)

$9(7.8)$

$69(60.0)$
$20(17.4)$
$15(13.0)$
$32(27.8)$
$5(4.4)$
$3(2.6)$

$56.8 \pm 7.7$

21 (18.3)

37 (32.2)

57 (49.6)

$48.8 \pm 6.4$

$13.0 \pm 31.0$

41 (35.7)

$0.8 \pm 0.3$

$11.7 \pm 5.9$

$2.6 \pm 0.5$

$42.8 \pm 8.2$

$45.6 \pm 20.3$

28 (24.4)

$9(7.8)$

21 (18.3)

17 (14.8)

$2(1.7)$

$1(0.9)$

$1(0.9)$

22 (19.1)

18 (15.7)

11 (9.6)

3 (2.6)

$1(0.9)$

3 (2.6)

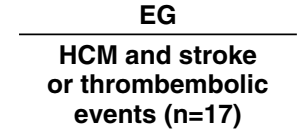

$60.0 \pm 9.7$

11 (64.7)

$2(11.8)$

5 (29.4)

12 (70.6)

$1(5.9)$

5 (29.4)

4 (23.5)

3 (17.6)

4 (23.5)

4 (23.5)

6 (35.3)

2 (11.8)

$1.2 \pm 1.6$

7 (41.2)

3 (17.7)

2 (11.8)

$13(76.5)$

3 (17.7)

5 (29.4)

5 (29.4)

$0(0.0)$

$0(0.0)$

$54.0 \pm 10.2$

2 (11.8)

5 (29.4)

$10(58.8)$

$50.2 \pm 5.5$

$15.9 \pm 28.8$

5 (29.4)

$0.9 \pm 0.2$

$13.2 \pm 5.5$

$2.3 \pm 0.4$

$46.9 \pm 7.5$

$57.9 \pm 17.3$

9 (52.9)

4 (23.5)

$6(35.3)$

$5(29.4)$

$0(0.0)$

$0(0.0)$

1 (5.9)

4 (23.5)

4 (23.5)

2 (11.8)

$0(0.0)$

1 (5.9)

1 (5.9)
NEG

$\begin{array}{cc}\begin{array}{c}\text { HCM without stroke } \\ \text { or thrombembolic } \\ \text { events }(\mathbf{n}=98)\end{array} & \text { P value } \\ 54.5 \pm 15.9 & 0.17 \\ 62(63.3) & 0.91 \\ 8(8.2) & 0.63 \\ & \\ 36(36.7) & 0.56 \\ 62(63.3) & 0.56 \\ 4(4.1) & 0.74 \\ 41(41.8) & 0.62 \\ 27(27.6) & 0.94 \\ 15(15.3) & 0.85\end{array}$

$11(11.2) \quad 0.17$

$21(21.4) \quad 0.87$

$27(27.6) \quad 0.80$

$7(7.1) \quad 0.52$

$\begin{array}{ll}7.1 \pm 1.2 & 0.79\end{array}$

$14(14.3) \quad<0.01^{\text {** }}$

$11(11.2) \quad 0.46$

$7(7.1) \quad 0.47$

$56(57.1) \quad 0.14$

$17(17.3) \quad 0.98$

$10(10.2) \quad 0.03^{\star}$

$27(27.6) \quad 0.88$

$5(5.1) \quad 0.35$

$3(3.1) \quad 0.47$

$\begin{array}{ll}57.3 \pm 7.0 & 0.12\end{array}$

$19(19.4) \quad 0.46$

$32(32.7) \quad 0.79$

$47(48.0) \quad 0.41$

$48.6 \pm 6.5 \quad 0.47$

$12.4 \pm 33.4 \quad 0.69$

$36(36.7) \quad 0.81$

$\begin{array}{ll}0.8 \pm 0.3 & 0.69\end{array}$

$11.5 \pm 6.0 \quad 0.49$

$2.6 \pm 0.5 \quad 0.08$

$41.7 \pm 8.0 \quad 0.02^{*}$

$42.5 \pm 19.7 \quad<0.01^{\star *}$

$19(19.4) \quad 0.01^{*}$

$5(5.1) \quad<0.01^{\star *}$

$15(15.3) \quad<0.05^{\star}$

$12(12.2) \quad 0.07$

$2(2.0) \quad 0.55$

$1(1.0) \quad 0.68$

$0(0.0) \quad 0.02^{*}$

$18(18.4) \quad 0.62$

$14(14.3) \quad 0.34$

$9(9.2) \quad 0.74$

$3(3.1) \quad 0.47$

$0(0.0) \quad 0.02^{*}$

$2(2.0) \quad 0.36$

(Table 1 continued the next page.) 


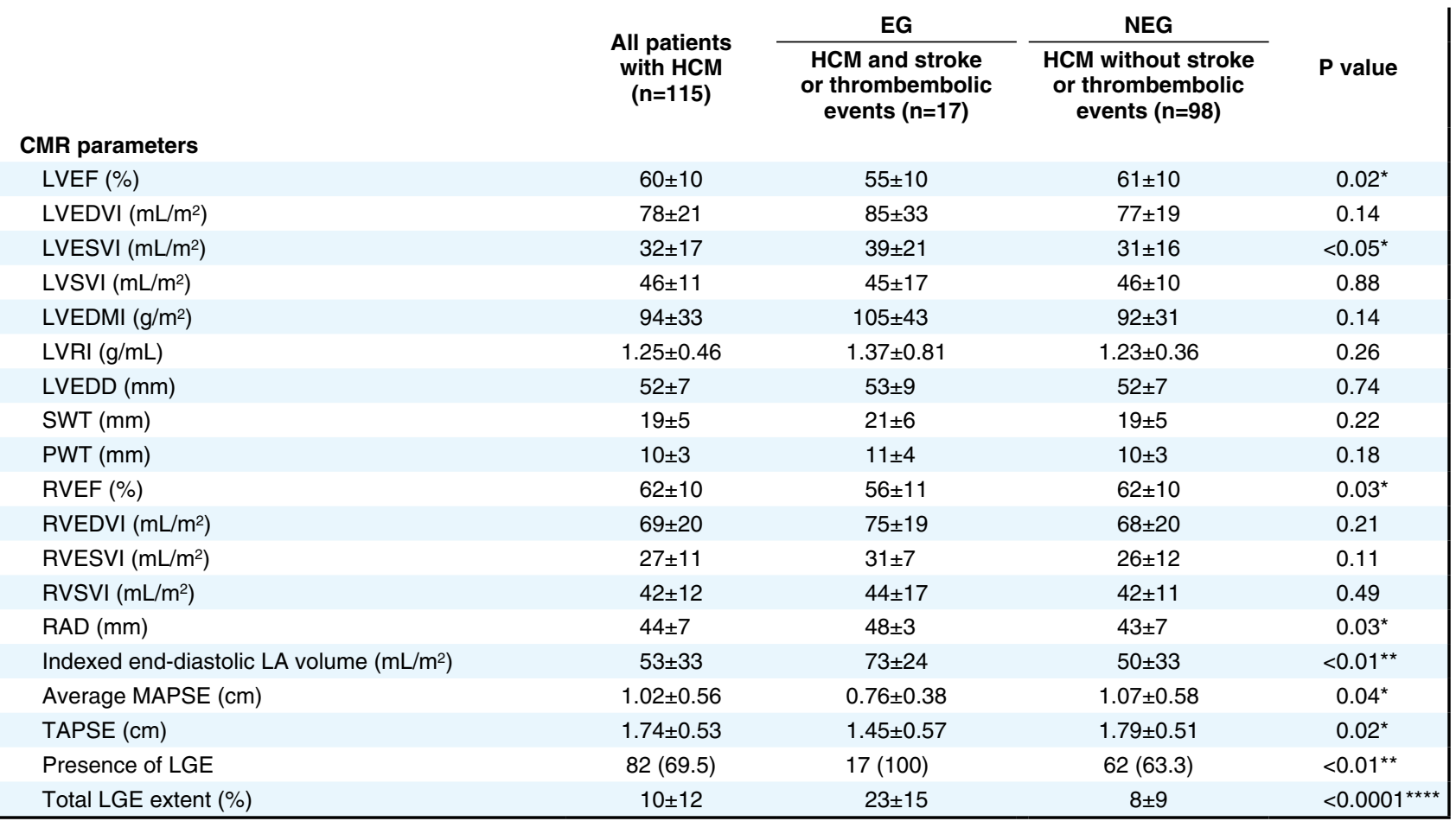

${ }^{A}$ Diastolic dysfunction defined as the presence of $\geq 2$ characteristics (E/e' $>14$, septal e' $<7 \mathrm{~cm} / \mathrm{s}$ or lateral e' $<10 \mathrm{~cm} / \mathrm{s}$, TR Vmax $>2.8 \mathrm{~m} / \mathrm{s}$, LAVI $>34 \mathrm{~mL} / \mathrm{m}^{2}$ ) according to Nagueh et al. ${ }^{51}$ Data are presented as the mean \pm standard deviation or number (\%) of subjects. Volumes are indexed to body surface area. Significance marked as ${ }^{\star} P<0.05,{ }^{* \star} P<0.01,{ }^{* \star *} P<0.001$, ${ }^{* \star \star *} P<0.0001$. EDD, end-diastolic dimension; EDMI, end-diastolic mass index; EDVI, end-diastolic volume index; ESVI, end-systolic volume index; HCM, hypertrophic cardiomyopathy; HNCM, hypertrophic nonobstructive cardiomyopathy; HOCM, hypertrophic obstructive cardiomyopathy; LA, left atrial; LGE, late gadolinium enhancement; LV, left ventricular; LVRI, left ventricular remodeling index; MAPSE, mitral annular plane systolic excursion; MI, myocardial infarction; PWT, posterior wall thickness; SCD, sudden cardiac death; SWT, septal wall thickness; VKA, vitamin K antagonist.

\begin{tabular}{|c|c|c|c|c|}
\hline \multirow{2}{*}{ Predictor } & \multicolumn{2}{|c|}{ Univariate } & \multicolumn{2}{|c|}{ Multivariate } \\
\hline & R square & $P$ value & OR $(95 \% \mathrm{Cl})$ & $P$ value \\
\hline LGE extent & 0.21 & $<0.0001$ & $1.1(1.05$ to 1.16$)$ & $<0.0001$ \\
\hline LAEDV & 0.06 & 0.0076 & $0.05(-0.01$ to 0.01$)$ & 0.19 \\
\hline $\mathrm{AF}$ & 0.06 & 0.0087 & 0.2 (0.05 to 0.72$)$ & 0.02 \\
\hline
\end{tabular}

$A F$, atrial fibrillation; $\mathrm{Cl}$, confidence interval; LAEDV, left atrial end-diastolic volume; LGE, late gadolinium enhancement; OR, odds ratio.

variables: LGE extent, LAEDV and presence of AF, proved to have $\mathrm{P}<0.01$ in the univariate analysis. To avoid overfitting, given the number of events, 2 variables were included at a time, LGE extent and LAEDV as a functional parameter or respectively LGE extent and presence of AF as a clinical parameter. Uniquely, the presence of LGE did not discriminate the group, because two-thirds of the patients without thromboembolic events had likewise LGE, which is why we chose the total extent of LGE instead. Furthermore, the size and volume of the LA provides prognostic information in patients with HCM, therefore LAEDV was included as a functional parameter. Since many patients of our study cohort suffered from AF, AF was included as a clinical parameter and a known risk factor for thromboembolic events.

In the first model (LGE extent and LAEDV), only LGE extent was found to be an independent predictor for thromboembolic events (odds ratio (OR) 1.1, CI [1.04-1.15],
$\mathrm{P}<0.0001)$. LAEDV did not reach statistical significance $(\mathrm{P}=0.19)$, so was not included in further analyses.

In the second model (LGE extent and AF), both LGE extent (OR 1.1, CI [1.05-1.16], P<0.0001) and AF (OR 0.2, CI $[0.05-0.72] \mathrm{P}=0.02)$ were independently associated with thromboembolic events, with a significantly stronger correlation for LGE extent.

Results of univariate and multivariate analyses are shown in Table 2.

ROC curves were used to find the optimal cutoff value for LGE extent (with maximizing of the sum of sensitivity and specificity) corresponding the most with the risk for thromboembolic events, and LGE extent $>14.4 \%$ (sensitivity $65 \%$, specificity $78.1 \%$; AUC $0.79, \mathrm{P}<0.0001$ ) was detected as threshold (Figure 1).

Based on the results of the multivariate and ROC analyses, a risk model was established, estimated with the KaplanMeier method and compared with the log-rank test. 


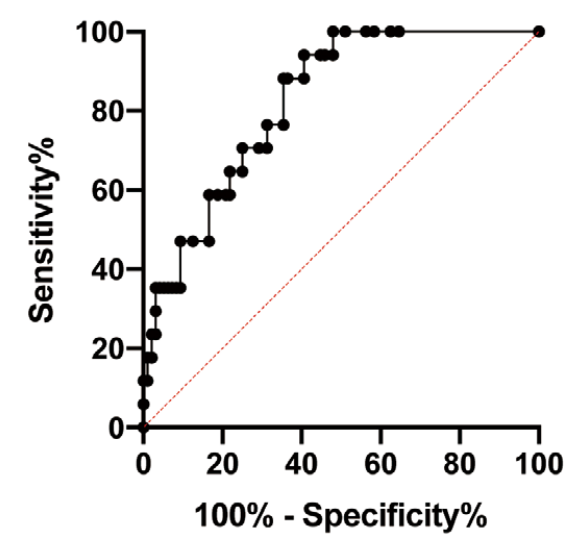

$p<0.0001$

AUC $0.82,95 \% \mathrm{Cl} 0.74$ to 0.91

Sensitivity 64.7

Specificity 78.1

Figure 1. Receiver-operating characteristic analysis for extent of late gadolinium enhancement (LGE, \%). AUC, area under the curve; $\mathrm{Cl}$, confidence interval.

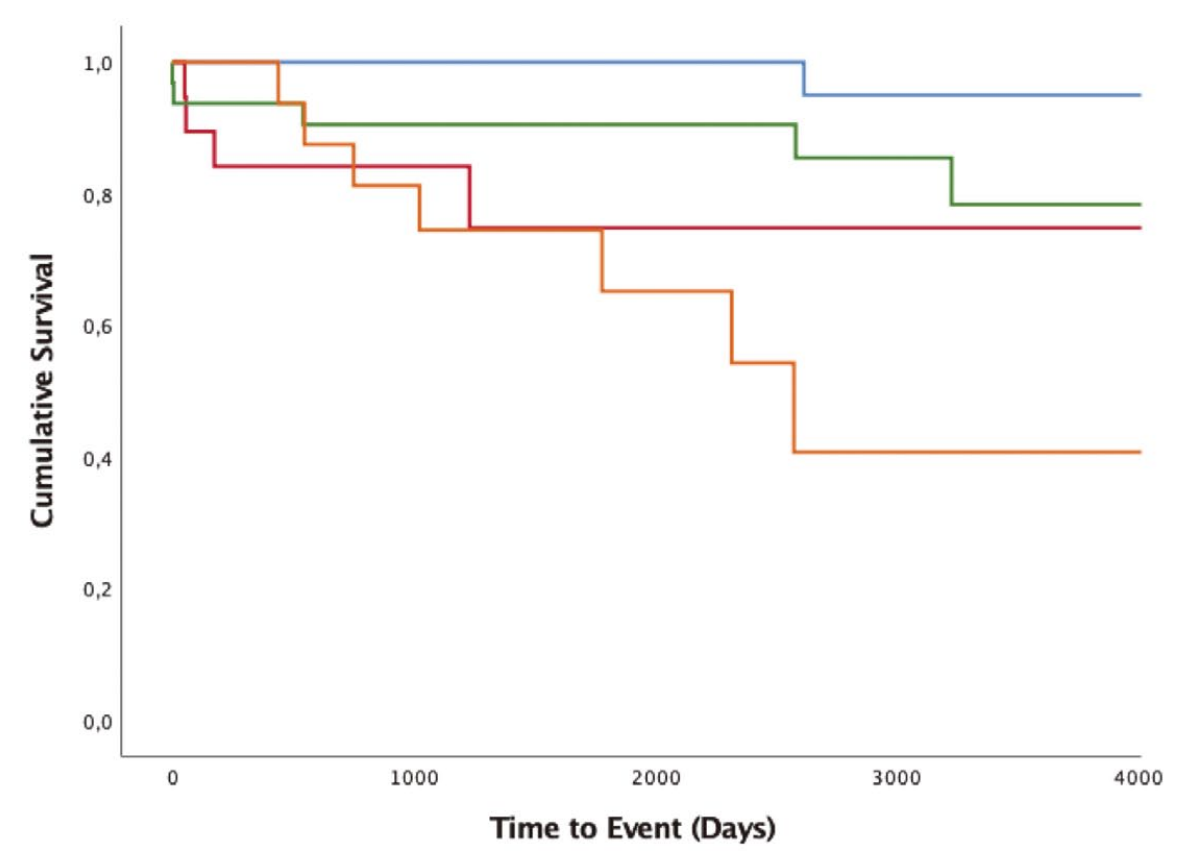

Groups

$\neg$ Absence of AF, LGE $\leq 14 \%$ $\neg$ Absence of AF, LGE > 14\% $\neg$ Presence of AF, LGE $\leq 14 \%$ $\neg$ Presence of AF, LGE > 14\%

Figure 2. Kaplan-Meier estimates according to the presence or absence of atrial fibrillation (AF) and LGE extent. Kaplan-Meier estimates were compared with the log-rank test. LGE, late gadolinium enhancement.

According to the presence or absence of AF and the extent of LGE, our study population was divided into 4 different patient groups. Patients with a combination of the 2 independent predictors (presence of AF and LGE extent $>14 \%$ ) had the highest risk for occurrence of thromboembolic events, which differed significantly from patients with a smaller LGE extent. There was no statistical significance regarding the 2 groups with LGE extent $>14 \%$, independent of the presence of AF.

The risk estimation model is shown in Figure 2.

\section{Discussion}

Despite advances in risk stratification, thromboembolic complications are still a considerable concern in patients with HCM. Therefore, we tried to ascertain predictors for these events in a HCM cohort by analyzing their clinical and CMR characteristics.

The main findings of the present study were: (1) the overall prevalence of thromboembolic events was high (17 of $115,14.8 \%$; (2) among patients who suffered the primary endpoint, AF was more frequently detected; (3) 4 of 17 patients $(23.5 \%)$ suffered a "cryptogenic" thromboembolic event without AF being identified as the underlying cause; (4) no patient without LGE experienced a thromboembolic event; and (5) LGE extent and the presence of AF were identified as independent predictors for thromboembolic events.

Thromboembolic events such as ischemic stroke and peripheral arterial thromboembolism are known complica- 
tions in patients with $\mathrm{HCM},{ }^{8}$ with prevalence varying from $6 \%$ to $23 \%,{ }^{7,8}$ which accords with the results of the present study.

Fibrosis as evaluated and measured by LGE in CMR is a hallmark in patients with HCM and reflects severity of the disease and is an independent predictor of major adverse events. ${ }^{16,17,31}$ LGE is a reliable, solid parameter, because detection is independent of, for example, hemodynamic parameters, volume state or heart rate. However, it is still unclear whether LA fibrosis emerges consequent to LV remodeling processes or if LA and LV fibrosis occur initially independently of each other or simultaneously and are manifestations of the same cardiomyopathic process. Because of limited spatial resolution it is difficult to accurately evaluate LGE imaging of the LA. Latif et al reported that in a small group of patients with HCM $(n=28)$, LA fibrosis correlated with the extent of LV LGE. ${ }^{32}$ Farhad et al detected that LA function was already impaired in HCM mutation carriers in the absence of pathognomonic LV hypertrophy, with further decline in patients with apparent HCM. ${ }^{33}$ These findings suggest that HCM is a cardiomyopathy that affects not only the ventricles but the atria as well, leading to an "atriomyopathy" with increased risk for thromboembolism. Because of this, thromboembolism can occur, even in the absence of AF. ${ }^{34}$ Thus, it can be supposed that LV fibrosis is very closely related to atrial fibrosis, either in the setting of coexisting processes and/or secondary through further deterioration of LA function/ fibrosis as a consequence of remodeling processes.

In our study cohort, 4 patients $(26.7 \%)$ developed thromboembolic events without detectable AF, although paroxysmal AF could not be ruled out for certain. However, in these 4 patients, LGE extent was considerably high. As we did not perform LGE analysis of the LA, we can only speculate about the extent of atrial fibrosis, but such speculation can be supported by the fact that these patients had considerable dilation and dysfunction of the LA, linking it to an atriomyopathy. These patients especially, who already show an advanced stage of the disease with greater extent of fibrosis in the LV but without detectable AF, should be followed up closely with periodic implementation of Holter monitoring. Larger, prospective studies are needed to investigate if patients with the described risk constellation (LGE extent $>14 \%$, no documented AF) should receive preventive treatment with anticoagulant drugs.

The majority of our study cohort $(79(68.7 \%))$ showed the presence of LGE. Thus, in our population no patient without LGE suffered a thromboembolic event. Because two-thirds of our patients without thromboembolic events had likewise LGE, uniquely the presence of LGE does not suffice as indicating a significant risk increase. In contrast, determination of the overall LGE extent seems to be a promising technique to detect patients who are at risk of developing thromboembolic events.

Enlargement of the LA is also a common finding in HCM and its size provides important prognostic information. ${ }^{35,36}$ Impaired LA function promotes AF, just as AF results in enlargement of the atria and diastolic dysfunction, finally culminating in a vicious circle. ${ }^{37,38}$ We performed LA volume assessment using the biplane area-length method, which can also be applied in patients with varying cardiac cycle length, as in AF. ${ }^{24}$ Increased LA volumes are strongly associated with the occurrence of AF in patients with HCM, ${ }^{39}$ and also an effective predictor for major adverse events (both cardiac and cerebrovascular events), ${ }^{40}$ even in patients with HCM without documented AF. ${ }^{35}$ Due to structural LA remodeling, AF in patients with HCM may reflect a more severe phenotype. ${ }^{41}$ However, the detection of paroxysmal AF is often an incidental finding, as patients are not continuously monitored.

Approximately $80 \%$ of our cohort received anticoagulant therapy, according to the ESC guidelines on diagnosis and management of $\mathrm{HCM},{ }^{42}$ which is in line with the experience in clinical practice. ${ }^{\mathbf{4 3}, \mathbf{4 4}}$ Although the ESC guidelines recommend anticoagulation with VKA, a number of patients received DOAC for stroke prevention. In patients receiving VKA the proportion of time in which the intensity of anticoagulation is in the therapeutic range ranges from $55 \%$ to $70 \%$, which may be the main limitation, as well as interactions with other drugs and food. ${ }^{4-47}$ All DOAC approval studies showed noninferiority to VKA and reduction of thromboembolic events such as ischemic stroke. 48-50 Furthermore, in a few cases of our cohort, the thromboembolic events occurred in patients who received anticoagulation therapy, but were not dosed sufficiently. This was noted more often in patients taking VKA, without reaching statistically significance. Nevertheless, data for anticoagulation with DOAC in patients with HCM are still lacking.

The present investigation demonstrated the additional benefit of LGE imaging for risk stratification in patients with HCM. Our findings emphasize the need for regular screening for AF, in particular in patients with the described risk constellation (LGE extent $>14 \%$ ), as approximately $25 \%$ of our cohort sustained a thromboembolic event without a known history of AF. Furthermore, the present results demand high awareness for AF, by both physicians and patients, because AF is strongly associated with thromboembolic events, especially in patients with HCM, indicating the benefit of anticoagulant therapy.

\section{Conclusions}

Myocardial fibrosis defined as LGE extent $>14 \%$ is an independent predictor for thromboembolic complications in patients with HCM and might therefore be considered as an important risk marker. The risk for thromboembolic events is significantly elevated if accompanied by AF, indicating the need for periodic ECG recording. Patients without detectable AF and the described risk constellation (LGE extent $>14 \%$ ) should be followed up closely, because patients with AF prophylactically receive anticoagulant therapy.

\section{Study Limitations}

Due to specific inclusion and exclusion criteria we reported a relatively small patient cohort of a single-center study. Multicenter studies are needed to confirm the present findings with potential clinical implications. Exclusion criteria may lead to a selection bias, as patients with ICD and severe renal dysfunction or previous stroke were excluded. In part of the study, follow-up was performed by telephone contact. Serial Holter monitoring was performed for AF screening at random times (at least twice per year), which guarantees no continuous detection and may underestimate the true incidence of (paroxysmal) AF. The described cutoff for LGE extent should not be considered as an absolute value, but rather taken in the context of other post-processing methods for quantification of the LGE extent, especially in patients with HCM, providing 
differing values; LGE quantification in our study was quantified by visual assessment. Attention should be paid to the fact that LGE was present in every patient who experienced a thromboembolic event, which should not mislead to the conclusion that patients without detectable fibrosis will not develop AF. Due to restricted image resolution, LGE analysis of the LA could not be performed.

\section{Competing Interests}

None.

\section{References}

1. Wigle ED, Rakowski H, Kimball BP, Williams WG. Hypertrophic cardiomyopathy: Clinical spectrum and treatment. Circulation 1995; 92: 1680-1692.

2. Maron BJ. Hypertrophic cardiomyopathy: A systematic review. JAMA 2002; 287: 1308-1320.

3. Maron BJ, McKenna WJ, Danielson GK, Kappenberger LJ, Kuhn HJ, Seidman CE, et al. American College of Cardiology/ European Society of Cardiology clinical expert consensus document on hypertrophic cardiomyopathy: A report of the American College of Cardiology Foundation Task Force on Clinical Expert Consensus Documents and the European Society of Cardiology Committee for Practice Guidelines. J Am Coll Cardiol 2003; 42: $1687-1713$.

4. Su M, Wang J, Kang L, Wang Y, Zou Y, Feng X, et al. Rare variants in genes encoding MuRF1 and MuRF2 are modifiers of hypertrophic cardiomyopathy. Int J Mol Sci 2014; 15: 9302-9313.

5. Elliott P, Andersson B, Arbustini E, Bilinska Z, Cecchi F, Charron $\mathrm{P}$, et al. Classification of the cardiomyopathies: A position statement from the European Society of Cardiology Working Group on Myocardial and Pericardial Diseases. Eur Heart J 2007; 29: 270-276.

6. Shigematsu Y, Hamada M, Mukai M, Matsuoka H, Sumimoto T, Hiwada K. Mechanism of atrial fibrillation and increased incidence of thromboembolism in patients with hypertrophic cardiomyopathy. Jpn Circ J 1995; 59: 329-336.

7. Higashikawa M, Nakamura Y, Yoshida M, Kinoshita M. Incidence of ischemic strokes in hypertrophic cardiomyopathy is markedly increased if complicated by atrial fibrillation. Jpn Circ J 1997; 61: 673-681.

8. Maron BJ, Olivotto I, Bellone P, Conte MR, Cecchi F, Flygenring $\mathrm{BP}$, et al. Clinical profile of stroke in 900 patients with hypertrophic cardiomyopathy. J Am Coll Cardiol 2002; 39: 301 - 307.

9. Olivotto I, Cecchi F, Casey SA, Dolara A, Traverse JH, Maron BJ. Impact of atrial fibrillation on the clinical course of hypertrophic cardiomyopathy. Circulation 2001; 104: 2517-2524.

10. Robinson K, Frenneaux MP, Stockins B, Karatasakis G, Poloniecki JD, Mckenna WJ. Atrial fibrillation in hypertrophie cardiomyopathy: A longitudinal study. J Am Coll Cardiol 1990; 15: $1279-1285$.

11. Guttmann OP, Rahman MS, O'Mahony C, Anastasakis A, Elliott PM. Atrial fibrillation and thromboembolism in patients with hypertrophic cardiomyopathy: Systematic review. Heart 2014; 100: 465-472.

12. Kirchhof P, Benussi S, Kotecha D, Ahlsson A, Atar D, Casadei B, et al. 2016 ESC Guidelines for the management of atrial fibrillation developed in collaboration with EACTS. Eur Heart $J$ 2016; 37: 2893-2962.

13. Authors/Task Force Members, Elliott PM, Anastasakis A, Borger MA, Borggrefe M, Cecchi F, Charron P, et al. 2014 ESC Guidelines on diagnosis and management of hypertrophic cardiomyopathy: The Task Force for the Diagnosis and Management of Hypertrophic Cardiomyopathy of the European Society of Cardiology (ESC). Eur Heart J 2014; 35: 2733-2779.

14. Moon JC, Reed E, Sheppard MN, Elkington AG, Ho S, Burke M, et al. The histologic basis of late gadolinium enhancement cardiovascular magnetic resonance in hypertrophic cardiomyopathy. $J$ Am Coll Cardiol 2004; 43: 2260-2264.

15. Rubinshtein R, Glockner JF, Ommen SR, Araoz PA, Ackerman $\mathrm{MJ}$, Sorajja P, et al. Characteristics and clinical significance of late gadolinium enhancement by contrast-enhanced magnetic resonance imaging in patients with hypertrophic cardiomyopathy. Circ Heart Fail 2010; 3: 51-58.

16. Bruder O, Wagner A, Jensen CJ, Schneider S, Ong P, Kispert EM, et al. Myocardial scar visualized by cardiovascular magnetic resonance imaging predicts major adverse events in patients with hypertrophic cardiomyopathy. J Am Coll Cardiol 2010; 56: 875887.

17. O'Hanlon R, Grasso A, Roughton M, Moon JC, Clark S, Wage R, et al. Prognostic significance of myocardial fibrosis in hypertrophic cardiomyopathy. J Am Coll Cardiol 2010; 56: 867-874.

18. Green JJ, Berger JS, Kramer CM, Salerno M. Prognostic value of late gadolinium enhancement in clinical outcomes for hypertrophic cardiomyopathy. JACC Cardiovasc Imaging 2012; 5: 370-377.

19. Doesch C, Tülümen E, Akin I, Rudic B, Kuschyk J, El-Battrawy I, et al. Incremental benefit of late gadolinium cardiac magnetic resonance imaging for risk stratification in patients with hypertrophic cardiomyopathy. Sci Rep 2017; 7: 6336.

20. Maron BJ, Towbin JA, Thiene G, Antzelevitch C, Corrado D, Arnett D, et al. Contemporary definitions and classification of the cardiomyopathies. Circulation 2006; 113: 1807-1816.

21. Simonetti OP, Kim RJ, Fieno DS, Hillenbrand HB, Wu E, Bundy JM, et al. An improved MR imaging technique for the visualization of myocardial infarction. Radiology 2001; 218: 215-223.

22. Papavassiliu T, Kühl HP, Schröder M, Süselbeck T, Bondarenko $\mathrm{O}$, Böhm CK, et al. Effect of endocardial trabeculae on left ventricular measurements and measurement reproducibility at cardiovascular MR imaging. Radiology 2005; 236: 57-64.

23. Alfakih K, Plein S, Thiele H, Jones T, Ridgway JP, Sivananthan MU. Normal human left and right ventricular dimensions for MRI as assessed by turbo gradient echo and steady-state free precession imaging sequences. J Magn Reson Imaging 2003; 17: $323-329$.

24. Sievers B, Kirchberg S, Addo M, Bakan A, Brandts B, Trappe HJ. Assessment of left atrial volumes in sinus rhythm and atrial fibrillation using the biplane area-length method and cardiovascular magnetic resonance imaging with TrueFISP. J Cardiovasc Magn Reson 2004; 6: $855-863$.

25. Pun SC, Figura M, Chow K, Haykowsky M, Thompson R, Paterson I. A simple method for characterizing left ventricular remodeling by cardiovascular magnetic resonance. $J$ Cardiovasc Magn Reson 2011; 13: P277.

26. Kramer CM, Barkhausen J, Flamm SD, Kim RJ, Nagel E. Standardized cardiovascular magnetic resonance imaging (CMR) protocols, Society for Cardiovascular Magnetic Resonance: Board of Trustees Task Force on standardized protocols. J Cardiovasc Magn Reson 2008; 10: 35.

27. Doesch C, Zompolou C, Streitner F, Haghi D, Schimpf R, Rudic $\mathrm{B}$, et al. CMR-derived TAPSE measurement: A semi-quantitative method of right ventricular function assessment in patients with hypertrophic cardiomyopathy. Neth Heart J 2014; 22: 557-564.

28. Spiewak M, Malek LA, Misko J, Chojnowska L, Milosz B, Klopotowski M, et al. Comparison of different quantification methods of late gadolinium enhancement in patients with hypertrophic cardiomyopathy. Eur J Radiol 2010; 74: e149-e153.

29. Doesch C, Huck S, Böhm CK, Michaely H, Fluechter S, Haghi $\mathrm{D}$, et al. Visual estimation of the extent of myocardial hyperenhancement on late gadolinium-enhanced CMR in patients with hypertrophic cardiomyopathy. Magn Reson Imaging 2010; 28: $812-819$.

30. Sacco RL, Adams R, Albers G, Alberts MJ, Benavente O, Furie $\mathrm{K}$, et al. Guidelines for prevention of stroke in patients with ischemic stroke or transient ischemic attack. Circulation 2006; 113: e409-e449.

31. Fortuni F, Angelini F, Abete R, Raineri C, Sclesi L, Turco A, et al. The prognostic value of late gadolinium enhancement in hypertrophic cardiomyopathy: An updated meta-analysis. Eur $J$ Prev Cardiol, doi:10.1177/2047487319874352.

32. Latif SR, Nguyen VQ, Peters DC, Soufer A, Henry ML, Grunseich $\mathrm{K}$, et al. Left atrial fibrosis correlates with extent of left ventricular myocardial delayed enhancement and left ventricular strain in hypertrophic cardiomyopathy. Int J Cardiovasc Imag 2019; 35: $1309-1318$

33. Farhad H, Seidelmann SB, Vigneault D, Abbasi SA, Yang E, Day SM, et al. Left Atrial structure and function in hypertrophic cardiomyopathy sarcomere mutation carriers with and without left ventricular hypertrophy. J Cardiovasc Magn Reson 2017; 19: 107.

34. Mac Grory B, Chang A, Atalay MK, Yaghi S. Left atrial appendage thrombus and embolic stroke: A case study. Stroke 2018; 49: e286-e289.

35. Haruki S, Minami Y, Hagiwara N. Stroke and embolic events in hypertrophic cardiomyopathy. Stroke 2016; 47: 936-942.

36. Minami Y, Haruki S, Yashiro B, Suzuki T, Ashihara K, Hagiwara 
N. Enlarged left atrium and sudden death risk in hypertrophic cardiomyopathy patients with or without atrial fibrillation. $J$ Cardiol 2016; 68: 478-484.

37. Tsang TS, Barnes ME, Gersh BJ, Bailey KR, Seward JB. Left atrial volume as a morphophysiologic expression of left ventricular diastolic dysfunction and relation to cardiovascular risk burden. Am J Cardiol 2002; 90: 1284-1289.

38. Pritchett AM, Mahoney DW, Jacobsen SJ, Rodeheffer RJ, Karon BL, Redfield MM. Diastolic dysfunction and left atrial volume: A population-based study. J Am Coll Cardiol 2005; 45: $87-92$.

39. Tani T, Tanabe K, Ono M, Yamaguchi K, Okada M, Sumida T, et al. Left atrial volume and the risk of paroxysmal atrial fibrillation in patients with hypertrophic cardiomyopathy. $J \mathrm{Am}$ Soc Echocardiogr 2004; 17: 644-648.

40. Tani T, Yagi T, Kitai T, Kim K, Nakamura H, Konda T, et al. Left atrial volume predicts adverse cardiac and cerebrovascular events in patients with hypertrophic cardiomyopathy. Cardiovasc Ultrasound 2011; 9: 34.

41. Sivalokanathan S, Zghaib T, Greenland GV, Vasquez N, Kudchadkar SM, Kontari E, et al. Hypertrophic cardiomyopathy patients with paroxysmal atrial fibrillation have a high burden of left atrial fibrosis by cardiac magnetic resonance imaging. JACC Clin Electrophysiol 2019; 5: 364-375.

42. Authors M. 2014 ESC Guidelines on diagnosis and management of hypertrophic cardiomyopathy: The Task Force for the Diagnosis and Management of Hypertrophic Cardiomyopathy of the European Society of Cardiology (ESC). Eur Heart $J$ 2014; 35: $2733-2779$.

43. Friberg L, Hammar N, Ringh M, Pettersson H, Rosenqvist M. Stroke prophylaxis in atrial fibrillation: Who gets it and who does not? Report from the Stockholm Cohort-study on Atrial Fibrillation (SCAF-study). Eur Heart J 2006; 27: 1954-1964.

44. Turakhia MP, Hoang DD, Xu X, Frayne S, Schmitt S, Yang F, et al. Differences and trends in stroke prevention anticoagulation in primary care vs. cardiology specialty management of new atrial fibrillation: The Retrospective Evaluation and Assessment of Therapies in AF (TREAT-AF) study. Am Heart J 2013; 165: 93-101.e101.
45. Erkens PM, ten Cate H, Büller HR, Prins MH. Benchmark for time in therapeutic range in venous thromboembolism: A systematic review and meta-analysis. PLoS One 2012; 7: e42269.

46. Gallego P, Roldán V, Marin F, Gálvez J, Valdés M, Vicente V, et al. SAMe-TT 2 R 2 score, time in therapeutic range, and outcomes in anticoagulated patients with atrial fibrillation. $\mathrm{Am}$ J Med 2014; 127: 1083-1088.

47. Ruff CT, Giugliano RP, Braunwald E, Hoffman EB, Deenadayalu $\mathrm{N}$, Ezekowitz MD, et al. Comparison of the efficacy and safety of new oral anticoagulants with warfarin in patients with atrial fibrillation: A meta-analysis of randomised trials. Lancet 2014; 383: $955-962$.

48. ROCKET AF Study Investigators. Rivaroxaban-once daily, oral, direct factor Xa inhibition compared with vitamin K antagonism for prevention of stroke and embolism trial in atrial fibrillation: Rationale and Design of the ROCKET AF study. Am Heart $J$ 2010; 159: 340-347.e341.

49. Lopes RD, Alexander JH, Al-Khatib SM, Ansell J, Diaz R, Easton JD, et al. Apixaban for reduction in stroke and other thromboembolic events in atrial fibrillation (ARISTOTLE) trial: Design and rationale. Am Heart J 2010; 159: 331-339.

50. Wallentin L, Yusuf S, Ezekowitz MD, Alings M, Flather M, Franzosi MG, et al. Efficacy and safety of dabigatran compared with warfarin at different levels of international normalised ratio control for stroke prevention in atrial fibrillation: An analysis of the RE-LY trial. Lancet 2010; 376: 975-983.

51. Nagueh SF, Smiseth OA, Appleton CP, Byrd BF 3rd, Dokainish $\mathrm{H}$, Edvardsen T, et al. Recommendations for the evaluation of left ventricular diastolic function by echocardiography: An update from the American Society of Echocardiography and the European Association of Cardiovascular Imaging. $J$ Am Soc Echocardiogr 2016; 29: 277-314.

\section{Supplementary Files}

Please find supplementary file(s);

http://dx.doi.org/10.1253/circj.CJ-19-0936 